\title{
Our distorted view of magnetars: application of the resonant cyclotron scattering model
}

\author{
Nanda Rea • Silvia Zane • Maxim Lyutikov • \\ Roberto Turolla
}

Received: 20 July 2006 / Accepted: 9 September 2006 / Published online: 15 March 2007

(C) Springer Science+Business Media B.V. 2007

\begin{abstract}
The X-ray spectra of the magnetar candidates are customarily fitted with an empirical, two component model: an absorbed blackbody and a power-law. However, the physical interpretation of these two spectral components is rarely discussed. It has been recently proposed that the presence of a hot plasma in the magnetosphere of highly magnetized neutron stars might distort, through efficient resonant cyclotron scattering, the thermal emission from the neutron star surface, resulting in production of non-thermal spectra. Here we discuss the Resonant Cyclotron Scattering (RCS) model, and present its XSPEC implementation, as well as preliminary results of its application to Anomalous X-ray Pulsars and Soft Gamma-ray Repeaters.
\end{abstract}

Keywords Neutron stars · Pulsars · Magnetars · X-ray · Resonant cyclotron scattering

N.R. is supported by an NWO Post-Doctoral Fellowship. S.Z. thanks the Particle Physics and Astronomy Research Council, PPARC, for support through an Advanced Fellowship.

\footnotetext{
N. Rea $(\varangle)$

SRON Netherlands Institute for Space Research, Sorbonnelaan, 2, 3584CA, Utrecht, The Netherlands

e-mail: N.Rea@sron.nl

S. Zane

Mullard Space Science Laboratory, University College of

London, Holbury St. Mary, Dorking Surrey, RH5 6NT, UK

\section{Lyutikov}

University of British Columbia, 6224 Agricultural Road,

Vancouver, BC, V6T 1Z1, Canada

R. Turolla

Physics Department, University of Padua, via Marzolo 8, 35131,

Padova, Italy
}

PACS 97.60.Jd $\cdot 97.60 . \mathrm{Gb}$

\section{Introduction}

Anomalous X-ray Pulsars (AXPs) and Soft Gamma-ray Repeaters (SGRs) are a small class of slowly rotating (5-12 s) neutron stars with emission properties much at variance with those of ordinary X-ray pulsars, both the young radio pulsars and the X-ray binary pulsars. They are called "anomalous" because their high X-ray luminosity $\left(10^{34}-10^{36} \mathrm{erg} / \mathrm{s}\right)$ cannot be easily explained in terms of the conventional processes which apply to other classes of pulsars, i.e. accretion from a binary companion or injection of rotational energy in the pulsar wind/magnetosphere. On the other hand, measurements of spin periods and period derivatives, when the latter are interpreted as due to electromagnetic dipolar losses, suggest that these objects may host "magnetars," i.e. neutron stars endowed with an ultra-strong magnetic field $\left(10^{14}-10^{15} \mathrm{G}\right.$, see Duncan and Thompson 1992; Thompson and Duncan 1993, 1996). The magnetar scenario appears so far very promising in explaining both the main energy source of these objects (the decay of the superstrong field) and the emission of the short, energetic bursts. Moreover, the magnetar scenario can account for the properties of Giant Flares, extremely energetic transient events ( $L \approx 10^{44}-10^{47} \mathrm{erg} / \mathrm{s}$ ) detected from SGRs. However, alternative scenarios to explain the enigmatic properties of these sources have been invoked. Among these, models involving accretion from a fossil disk, left by the supernova event which gave birth to the neutron star, are still largely plausible (van Paradijs et al. 1995; Chatterjee et al. 2000). Very recently such a (maybe passive) disk has been indeed observed in the IR around AXP 4U $0142+61$ (Wang et al. 2006). Magnetars candidates are strong X-ray sources, and their 
$\mathrm{X}$-ray spectra in the $0.5-10 \mathrm{keV}$ band are usually fit with a two component model consisting of (besides interstellar absorption) a thermal blackbody with a typical temperature $k T \sim 0.3-0.4 \mathrm{keV}$ and a power-law with a relatively steep photon index, $\Gamma \sim 2-4$ (see Kaspi 2006 in this volume, and Woods and Thompson 2004 for recent reviews). In some cases, SGR spectra have been fit with a single power-law component, but recent longer observations showed that, also for these sources, a blackbody component is often required (Mereghetti et al. 2005a, 2005b).

Despite the fact that the blackbody plus power-law spectral model has been largely applied to magnetar spectra for many years, a reliable physical interpretation of these two components is still missing. Quite recently, an attempt to interpret the observed spectrum and long term variations of 1E 1048.1-5937 in terms of a (non-resonant) Comptonization model has been presented by Tiengo et al. (2005). Furthermore, the recent discovery of magnetar counterparts in the radio to the $\gamma$-ray bands (Camilo et al. 2006; Hulleman et al. 2000; Kuiper et al. 2004) enforced the idea that their multiwavelength spectral energy distribution is by far more complicated than a simple blackbody plus power-law distribution (see also Den Hartog et al. contribution in this volume).

\section{The Resonant Cyclotron Scattering (RCS) model applied to magnetars spectra}

Following the original suggestion by Thompson et al. (2002), it has been proposed by Lyutikov and Gavriil (2006) that the presence of a warm non-relativistic plasma in the magnetosphere of magnetars, might be responsible, through efficient resonant electron cyclotron scattering, of distorting the thermal X-ray emission from the star surface. In particular, if a large volume of the neutron star magnetosphere of the is partially filled by $e^{ \pm}$-currents, the thermal (or quasithermal) cooling radiation emerging from the star surface will experience repeated scatterings at the cyclotron resonance.

The efficiency of the process is quantified by the scattering optical depth, $\tau_{\text {res }}$. Following again Lyutikov and Gavriil (2006), and assuming a dipolar configuration for the magnetic field, this can be written as

$\tau_{\text {res }}=\int \sigma_{\text {res }} n d l=\tau_{0}\left(1+\cos ^{2} \alpha\right)$

where $\sigma_{\text {res }}$ is the cross section for electron scattering in the magnetized regime, $n$ is the electrons number density, $\alpha$ is the angle between the photon propagation direction and the local magnetic field, and

$\tau_{0}=\frac{\pi^{2} e^{2} n r}{3 m_{e} c \omega_{B}}$.
Here $r$ is the radial coordinate, $\omega_{B}=e B / m_{e} c$ is the electron cyclotron frequency, and $B$ is the local value of the magnetic field. At variance with the case of unmagnetized Thomson scattering, in presence of a magnetic field $\sigma_{\text {res }}$ is resonant and is given by

$\sigma_{\text {res }}=\frac{\sigma_{T}}{4} \frac{\left(1+\cos ^{2} \alpha\right) \omega^{2}}{\left(\omega-\omega_{B}\right)^{2}+\Gamma^{2} / 4}$

where $\Gamma=4 e^{2} \omega_{B}^{2} / 3 m_{e} c^{3}$ is the natural width of the first cyclotron harmonic and $\sigma_{T}$ is the cross-section for unmagnetized Thomson scattering. The crucial point is that, in this model, the plasma distribution is spatially extended. Since the value of the resonant energy depends on the local value of the magnetic field, repeated scatterings of photons could lead to the formation of a high energy tail instead of a narrow line. At relatively large distances from the surface, the magnetic field drops to typical values such that scattering is resonant for soft X-ray photons, below $\sim 10 \mathrm{keV}$. On the other hand, at these energies the resonant scattering optical depth greatly exceeds that for Thomson scattering, $\tau_{T} \sim r n \sigma_{T}$,

$$
\frac{\tau_{\mathrm{res}}}{\tau_{T}} \sim \frac{\pi}{8} \frac{c}{r_{e} \omega_{B}} \sim 10^{5}\left(\frac{1 \mathrm{keV}}{\hbar \omega_{B}}\right)
$$

where $r_{e}=e^{2} / m c^{2}$ is the classical electron radius. This implies that even a relatively small amount of plasma suspended in the magnetosphere of the neutron star may considerably modify the emergent spectra. There is now increasing support to the idea that the magnetar magnetospheres might be twisted (Thompson et al. 2002), in which case they may be partially filled by $e^{-}$currents with densities well in excess of the Goldreich-Julian density (which is expected in the case of a simple dipolar configuration, see Goldreich and Julian 1969).

A further effect is related to the fact that the spatial region, over which such currents are distributed, is permeated by a highly inhomogeneous magnetic field. Although for soft X-ray photons the scattering conserves energy in the electron rest frame, thermal/bulk electron motion produces an energy transfer in the observer rest frame. Multiple resonant cyclotron scatterings of thermal photons will, on average, up-scatter the transmitted radiation, resulting in the formation of a non-thermal spectrum. The emerging spectrum will then appear distorted and, as it is the case for thermal non-magnetic comptonization, its shape may be well represented by the superposition of thermal and non-thermal components (see Fig. 1).

Here we present an application of these models to magnetar spectra. Theoretical models are computed as in Lyutikov and Gavriil (2006), under the assumption that scattering occurs in a non-relativistic, static, warm medium and neglecting electron recoil, i.e. in the limit $\beta_{t h}<1$ (where $\beta_{t h}$, a model parameter, is the thermal velocity of electrons 


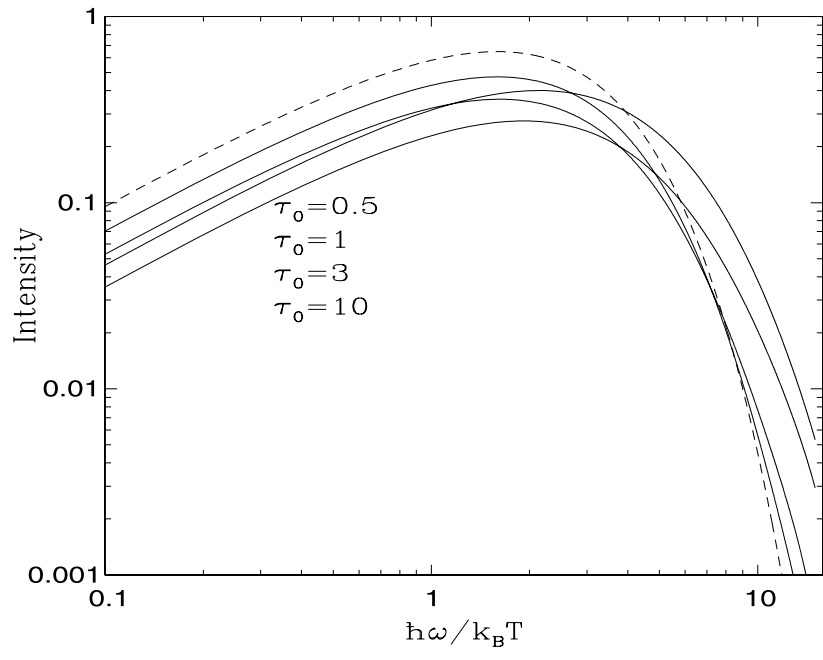

Fig. 1 Modification of an initially Plankian spectrum (dashed line) by multiple cyclotron scattering for different values of $\tau_{0}$ and $\beta_{t h}=0.3$ (Lyutikov and Gavriil 2006)

in units of the speed of light) and for low-energy photons, $\epsilon \ll m c^{2}$. Within the range of parameter values allowed by the numerical code, the non-thermal effects are most prominent for models in which the plasma is mildly relativistic, $\beta_{t h} \lesssim 1$, and the optical depth is reaching its boundary value $\tau_{\text {res }} \sim 10$.

\section{The RCS XSPEC model}

In order to perform a quantitative comparison between fits to magnetar spectra performed with the model described above and with the canonical blackbody plus power-law model, we developed a code to upload the RCS model into XSPEC. We created a grid of intensity tables (through a Monte-Carlo simulation) for a set of values of the three model parameters, i.e. $\beta_{t h}, \tau_{0}$ and $T$, where $T$ is the temperature of the seed thermal surface emission (assumed to be a blackbody). The parameters ranges used were $0.1<\beta_{t h}<0.5$ (step 0.1), $1<\tau<10$ (step 1) and $0.1 \mathrm{keV}<T<3 \mathrm{keV}$ (step $0.05 \mathrm{keV}$ ). For each model, the spectrum has been computed in the energy range $0.1-12 \mathrm{keV}$ (step $0.05 \mathrm{keV}$ ). The final XSPEC atable spectral model has therefore four parameters, the three listed above plus the last one being the normalization constant, which are simultaneously varied during the spectral fitting following the standard $\chi^{2}$ minimization technique.

Note that a previous attempt to apply the Lyutikov and Gavriil (2006) model to spectral data was presented by the authors themselves. However, in that case $\beta_{t h}$ and $\tau_{0}$ were fixed a priori during each fit and eventually varied manually. The fit itself was done only by varying $T$ and the normalization factor, while in this case we are able to fit simultaneously all the four parameters, resulting in a more precise
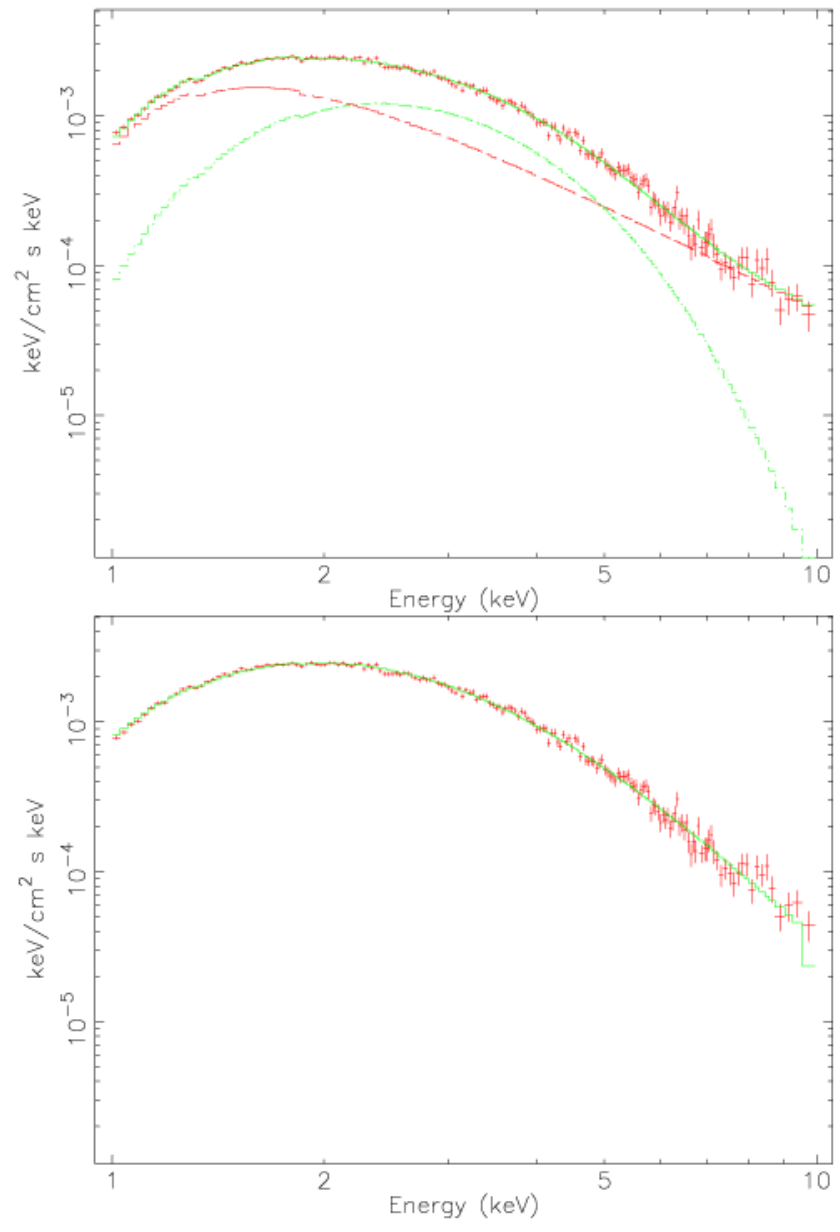

Fig. 2 The XMM-Newton spectrum of 1E 1048-5937 fitted with an absorbed power-law plus a blackbody (top panel) and with the RCS model (bottom panel)

determination of their best fitting values and in a more reliable $\chi^{2}$ determination.

\section{Preliminary results}

Here we present the preliminary results of the spectral modelling of magnetars with the Resonant Cyclotron Scattering model (RCS). We concentrate on the soft X-ray spectra (1-10 keV) of three sources, 1E 1048-56, 1RXS J17084009 and SGR 1806-20, and we use data obtained with the XMM-Newton satellite. Detailed information about the observations, data reduction and analysis can be found in Mereghetti et al. (2004) and Rea et al. (2005a, 2005b), for the three sources, respectively.

The fit of the X-ray spectrum of 1E 1048-56 is presented in Fig. 2 and Table 1. In this case we find that the RCS model is successful in reproducing the data, as well as the canonical blackbody plus power-law model (BB+PL). The two fits have the same number of degrees of freedom. The 
Table 1 Best fit values of the spectral parameters obtained by fitting the XMM-Newton spectrum of 1E 1048-5937 with the BB+PL and an RCS model (for more details about this observation see Mereghetti et al. (2004)). Errors are at $1 \sigma$ confidence level, and the reported flux is absorbed and in the $2-10 \mathrm{keV}$ energy range

\begin{tabular}{lll}
\hline & BB + PL & RCS model \\
\hline $\mathrm{N}_{H}\left(10^{22} \mathrm{~cm}^{-2}\right)$ & $1.12_{-0.02}^{+0.04}$ & $0.49_{-0.02}^{+0.01}$ \\
$\mathrm{kT}(\mathrm{keV})$ & $0.64_{-0.01}^{+0.01}$ & $0.53_{-0.01}^{+0.01}$ \\
$\mathrm{BB}$ norm & $1.03_{-0.01}^{+0.02} \times 10^{-4}$ & - \\
$\Gamma$ & $3.3_{-0.1}^{+0.2}$ & - \\
$\mathrm{PL}$ norm & $1.10_{-0.04}^{+0.13} \times 10^{-2}$ & - \\
$\beta_{t h}$ & - & $0.41_{-0.02}^{+0.01}$ \\
$\tau_{0}$ & - & $1.9_{-0.1}^{+0.1}$ \\
RCS norm & - & $0.22_{-0.03}^{+0.01}$ \\
$\chi_{v}^{2}$ & 0.99 & 1.08 \\
Flux $\left(\mathrm{erg} \mathrm{s}^{-1} \mathrm{~cm}^{-2}\right)$ & $7.9_{-0.1}^{+0.1} \times 10^{-12}$ & $7.9_{-1.7}^{+1.5} \times 10^{-12}$ \\
\hline
\end{tabular}

value of the column density found with the RCS model is lower (although both consistent with Durant and van Kerkwijk 2006), while the temperature of the thermal component $T$ and the total flux are consistent with that found with the $\mathrm{BB}+\mathrm{PL}$ modelling. These results are in agreement with the previous findings by Lyutikov and Gavriil (2006), who presented a similar attempt by using Chandra data and varying manually some of the model parameters to reproduce the spectrum.

As mentioned before, the technique used here is more reliable in providing a determination of the best fitting parameters. In this case, the source spectrum can be reproduced by invoking scattering by $e^{-}$with thermal velocity $\beta_{t h}=0.41$ and optical depth $\tau_{0}=1.9$.

On the other hand, the cases of 1RXS J1708-4009 and SGR 1806-20 are more problematic. These sources have much harder X-ray spectra (in the $1-10 \mathrm{keV}$ band), that cannot be accounted for by the RCS model alone (see Fig. 3) at least when the spectral deformation resulting from the resonant scattering process is computed in the simple way proposed by Lyutikov and Gavriil (2006).

However, it is worth noting that, at variance with $1 \mathrm{E}$ 1048-56, these two sources exhibit strong high-energy power-law tails which extend up to the $\gamma$-rays (Kuiper et al. 2006; Molkov et al. 2005; Mereghetti et al. 2005a). Motivated by that, we fitted the whole $1-50 \mathrm{keV}$ spectra with the RCS model plus a power-law. For both sources, we obtained a good fit with a reduced $\chi^{2}$ of 1.08 and 1.1 , respectively. Although extremely promising, these analysis are very preliminary and they still require a careful assessment of intercalibration issues which arise when different detectors are used. Therefore, we do not discuss the results in more detail nor we present here best fitting parameter values. These results will be presented in more de-
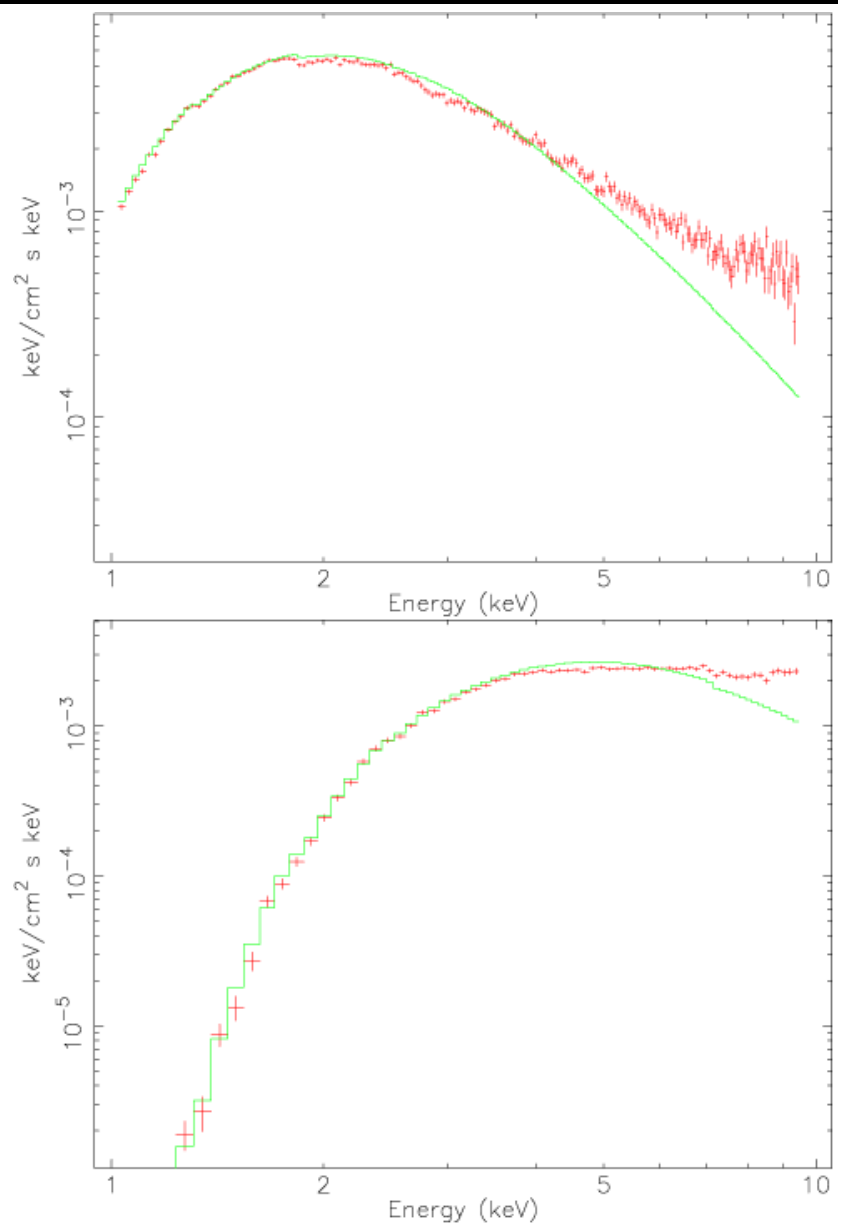

Fig. 3 XMM-Newton spectra of 1RXS J1708-4009 (top panel) and SGR 1806-20 (bottom panel) fitted with he RCS model. The black-body plus power-law fits might be found in Rea et al. (2005a) and Mereghetti et al. (2005a, 2005b)

tails in a forthcoming paper (Rea et al. 2006, in preparation).

\section{Conclusion}

The preliminary results reported here show that the X-ray spectra of the AXP 1E 1048-56 can be successfully reproduced by the RCS model. The quality of the fit is comparable to that obtained by using the canonical blackbody plus power-law model, with the advantage that the RCS model spectra provide a better physical understanding of the presence of the thermal/non-thermal components in the observed spectrum. On the other hand, this is not totally unexpected since this source has a non-thermal tail which is relatively soft with respect to other AXPs, and a similar result has already been found by Lyutikov and Gavriil, although their fitting technique was less accurate. For the other two sources considered here, 1RXS J1708-4009 and SGR 1806-20, we find that the RCS model alone cannot account 
for the relatively hard non-thermal component observed below $\sim 10 \mathrm{keV}$.

On the other hand, in both cases a composite model consisting of a RCS model plus a power-law can fit the X-ray spectra in the $1-100 \mathrm{keV}$ band. This may suggest that, in the 6-10 keV energy range, the X-ray emission of these sources is contaminated by the hard non-thermal component which has been recently observed with INTEGRAL at much higher energies (Kuiper et al. 2006). A similar hard tail has not been observed, at least so far, in the case of 1E 1048-56. A detailed paper with a systematic application of the RCS model to the whole class of magnetars and a study of their spectral and flux variability in this scenario is in preparation, accompanied by a more complete discussion (Rea et al. 2006, in preparation).

Acknowledgements We thank Valentina Bianchin and Gavin Ramsay for their kind help in building the XSPEC RCS model. We also thank Fotis Gavriil for having kindly allowed us to look into his preliminary model, Andrea Tiengo, Gianluca Israel and the anonymous referee for useful comments. NR thanks the Mullard Space Science Laboratory, where this work was partially done, for the warm hospitality.

\section{References}

Camilo, F., Ransom, S., Halpern, J., et al.: Nature 442(7105), 892-895 (2006) Preprint (astro-ph/0605429)

Chatterjee, P., Hernquist, L., Narayan, R.: Astrophys. J. 534, 373 (2000)
Duncan, R.C., Thompson, C.: Astrophys. J. 392, L9 (1992)

Durant, M., van Kerkwijk, M.H.: Astrophys. J. 650, 1070 (2006) (astro-ph/0606604)

Goldreich, P., Julian, W.H.: Astrophys. J. 157, 869 (1969)

Hulleman, F., van Kerkwijk, M.H., Kulkarni, S.R.: Nature 408, 689 (2000)

Kuiper, L., Hermsen, W., Mendez, M.: Astrophys. J. 613, 1173 (2004)

Kuiper, L., Hermsen, W., den Hartog, P.R., Collmar, W.: Astrophys. J. 645, 556 (2006)

Lyutikov, M., Gavriil, F.: Mon. Not. Roy. Astron. Soc. 368, 690 (2006)

Mereghetti, S., Tiengo, A., Stella, L., et al.: Astrophys. J. 608, 427 (2004)

Mereghetti, S., Tiengo, A., Esposito, P., et al.: Astrophys. J. 628, 938 (2005a)

Mereghetti, S., Gotz, D., Mirabel, I.F., Hurley, K.: Astron. Astrophys. 433, L9 (2005b)

Molkov, S., Hurley, K., Sunyaev, R., et al.: Astron. Astrophys. 433, L13 (2005)

Rea, N., Oosterbroek, T., Zane, S., et al.: Mon. Not. Roy. Astron. Soc. 361, 710 (2005a)

Rea, N., Israel, G.L., Covino, S., et al.: Astron. Telegr., \#645 (2005b)

Thompson, C., Duncan, R.C.: Astrophys. J. 408, 194 (1993)

Thompson, C., Duncan, R.C.: Astrophys. J. 473, 322 (1996)

Thompson, C., Lyutikov, M., Kulkarni, S.R.: Astrophys. J. 574, 332 (2002)

Tiengo, A., Mereghetti, S., Turolla, R., et al.: Astron. Astrophys. 437, 997 (2005)

van Paradijs, J., Taam, R.E., van den Heuvel, E.: Astron. Astrophys. 299, L41 (1995)

Wang, Z., Chakrabarty, D., Kaplan, D.L.: Nature 440(7085), 772-775 (2006)

Woods, P.M., Thompson, C.: Preprint (2004) (astro-ph/0406133) 